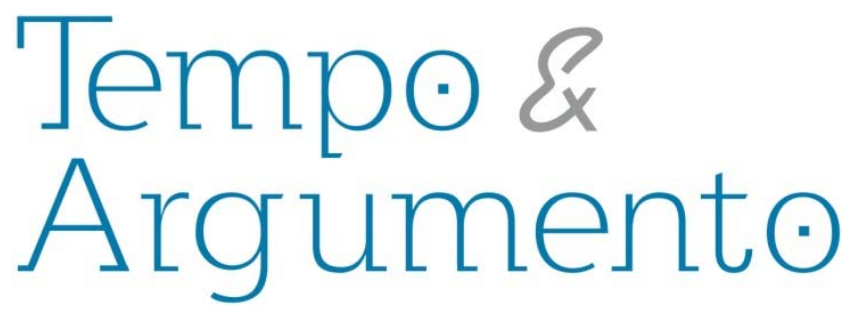

\title{
La Historia local en tiempos de Internet. Nuevos cauces para una especialización disciplinaria
}

\begin{abstract}
Resumen
Los procesos de globalización, potenciados por las redes telemáticas, estimulan y al mismo tiempo desafían a las identidades locales en grados insospechados. La Historia local -antigua práctica disciplinaria transformada en una especialización científico-social- refleja, en sus desarrollos recientes, los cambios que experimenta su objeto de estudio, incorporando una variada gama de recursos, herramientas y aplicaciones que le proporcionan las nuevas tecnologías. Ya sea en la labor de relevamiento bibliográficodocumental, en el análisis de la información que procede de las fuentes, o en la presentación de los resultados obtenidos en sus investigaciones, los historiadores locales del siglo XXI se sirven de Internet de manera sistemática. A partir de la evidencia que aportan ciertos casos, este artículo reflexiona, desde una perspectiva metodológica, sobre las modalidades que asume el vínculo entre los estudios del pasado local y los recursos que brinda "la red de redes".
\end{abstract}

Palabras clave: Historia Local - Internet - Metodología.

\author{
Juan Andrés Bresciano Lacava \\ Doctor en Historia por la Facultad de \\ Filosofía y Letras de la Universidad de \\ Buenos Aires. Profesor Agregado, Grado 4 \\ del Departamento de Historiología, Instituto \\ de Ciencias Históricas, de la Facultad de \\ Humanidades y Ciencias de la Educación de \\ la Universidad de la República \\ (Montevideo, Uruguay). \\ jabresciano@fhuce.edu.uy
}

\section{Para citar este artículo:}

BRESCIANO, Juan Andrés. La Historia local en tiempos de Internet. Nuevos cauces para una especialización disciplinaria. Revista Tempo e Argumento, Florianópolis, v. 6, n. 12, p. 05 - 22, mai./ago. 2014.

\section{DOI: $10.5965 / 2175180306122014005$}

http://dx.doi.org/10.5965/2175180306122014005 


\title{
History in times of internet. New channels for a disciplinary specialization
}

\begin{abstract}
The globalization processes, enhanced by telematic networks, stimulate and at the same time challenge local identities at unsuspected levels. Local History old disciplinary practice turned into a scientific-social specialization - reflects, on its recent developments, the changes experienced by its study object, incorporating a wide range of resources, tools, and applications it is provided with by the new technologies. Either through the work of bibliographical and documentary survey, the analyses of information derived from sources, or the presentation of results obtained by means of their investigation, $21^{\text {st }}$ century local historians use Internet systematically. Based on the evidence provided by certain cases, this article reflects, from a methodological perspective, on modalities taken by the link between studies addressing the local past and the resources offered by the "network of networks".
\end{abstract}

Keywords: Local History - Internet - Methodology.

\section{Introducción}

Durante la última década del siglo XX, las tecnologías de la información y de la comunicación contribuyeron a redefinir las fronteras y las escalas de la interacción humana. El desarrollo de redes telemáticas que desterritorializan los vínculos sociales, la conformación de un ciberespacio que se nutre localmente y se despliega mundialmente, y la cristalización de una cultura multimediática que introduce nuevos códigos comunicativos incentivan el fenómeno tan peculiar de la glocalización. Este término refiere a las adaptaciones locales de modalidades 
operativas y de recursos simbólicos procedentes del contexto mundial, que revitalizan las pequeñas comunidades y las proyecta con renovado vigor más allá de los límites en que solían desenvolverse (Robertson, 1992). Al desdibujar las fronteras geográficas y resignificar las identidades residenciales (Castells, 1996-1998), las nuevas tecnologías brindan la posibilidad de que tanto investigadores como ciudadanos corrientes las utilicen para una comprensión más profunda de sus realidades inmediatas y las empleen para preservar y difundir, en ámbitos plurales, el patrimonio histórico-cultural de los espacios a los que pertenecen.

En este contexto, la Historia local (entendida como una expresión científico-social y no como el cultivo erudito de un saber de estrechos horizontes) experimenta una significativa renovación, según lo demuestran diversas obras emblemáticas aparecidas en la pasada década (Amato [2002]; Kammen [2003]; Brooks [2008]; Kyvig y Marty [2010]). Gracias a la revolución digital, esa renovación se plasma en una ampliación del campo disciplinario y en la reformulación de las prácticas heurísticas y hermenéuticas (Dyer et al., 2011). Por tal motivo, el presente artículo se propone determinar el modo en que Internet gravita en las tres instancias cruciales del desarrollo de las investigaciones histórico-locales: la fase del relevamiento bibliográficodocumental, la fase del procesamiento y análisis de datos, y la fase de presentación y divulgación de los resultados. En cada una de estas instancias, las referencias a ejemplos específicos de procedencia diversa ilustrarán algunas constataciones formuladas desde la reflexión teórica.

\section{La ampliación del espectro heurístico}

En los últimos veinte años, la difusión de dispositivos que permiten digitalizar fuentes, la aparición de sitios web que difunden voluminosas colecciones documentales, y la multiplicación de programas y utilitarios que facilitan el análisis de toda clase de contenidos, modifican los parámetros en los que se desenvolvía tradicionalmente el historiador en sus labores heurísticas (Bresciano, 2010). Operando de manera conjunta o autónoma, esos recursos afectan al modo en que, en la escala local, el investigador aborda los diversos tipos de registros: los textuales, los gráficos, los iconográficos, los orales, los sonoros y los audiovisuales.

\subsection{Las fuentes textuales electrónicas}

En cuanto insumos característicos de las pesquisas históricas, los materiales bibliográficos, los artículos de prensa y los documentos de archivo resultan mucho más accesibles gracias a las 
ediciones electrónicas y los proyectos de digitalización masiva impulsados por cientos de bibliotecas y archivos en decenas de países (Bunz, 2014). Sin lugar a dudas, emprendimientos de esta índole inciden, de manera diferencial, en la utilización de libros, artículos y documentos para los estudios sobre el pasado comunitario.

\subsubsection{Las fuentes bibliográficas}

Las obras que se refieren a una localidad o ciudad determinada (o que la analizan desde una perspectiva diacrónica) conforman un vasto universo, heterogéneo y disperso, distribuido en numerosas bibliotecas. En no pocas ocasiones, libros y folletos de gran relevancia para el estudio de un pueblo o de una región se encuentran disponibles exclusivamente en bibliotecas extranjeras. Por ello, la progresiva digitalización de las colecciones de los repositorios bibliográficos mundiales cumple un papel fundamental en la difusión de textos que trascienden por sus contenidos - las fronteras políticas. Asimismo, la publicación electrónica de obras de aparición reciente ${ }^{1}$ favorece la incorporación de materiales que no resultarían de fácil obtención. De hecho, el desarrollo de compañías editoriales que utilizan exclusivamente el soporte digital no sólo amplía el campo de divulgación de bibliografía local, sino que genera un fenómeno cultural potencialmente revolucionario, al que el historiador de las próximas décadas deberá habituarse. ${ }^{2}$

\subsubsection{Las fuentes hemerográficas}

Los órganos de prensa en todas sus variantes configuran medios privilegiados para el estudio del pasado. Por la información fáctica que proporcionan sobre acontecimientos específicos y por las opiniones individuales y colectivas que difunden, testimonian aspectos sustanciales del devenir comunitario, en las dimensiones fundamentales de la vida pública, y en algunas de la vida privada. Dado que las nuevas tecnologías transforman las capacidades comunicativas de los medios de prensa y abaratan los costos de edición, numerosos periódicos locales que corrían el riesgo de desaparecer adquieren un inusitado vigor, mientras que surgen otros nuevos que sólo se publican en formato electrónico (Patel, 2010). La prensa barrial o municipal, lejos de desaparecer ante la fuerza avasalladora de la globalización, se convierte en el pilar de identidades florecientes, que difunden sus inquietudes, demandas e intereses en espacios que no se restringen a los canales tradicionales de circulación. De este modo, las tecnologías de la

\footnotetext{
${ }^{1}$ Los servicios que ofrece Questia.com (2014) ilustran ampliamente esta modalidad.

${ }^{2}$ Un ejemplo reciente de esto lo aporta Online Books (2014).
} 
información diluyen las fronteras entre lo local y lo mundial, mediante diferentes grados de interdependencia.

\subsubsection{Las fuentes telemáticas}

El crecimiento exponencial de Internet en los años noventa, modifica las formas en que se estructuran, difunden y conservan los materiales relacionados con el estudio del pasado local. Sin lugar a dudas, la emergencia de los sitios web como componentes primarios de la red promueve el desarrollo, potencialmente ilimitado, de ciertas familias tipológico-documentales, mediante las que se canalizan las expresiones políticas, sociales, económicas y culturales de barrios, municipios o regiones. Surge, entonces, un corpus específico de documentos electrónicos que se convierte en un referente insoslayable para cualquier investigador presente o futuro. Integrado por miles de sitios institucionales o personales, que reflejan o manifiestan las realidades y las problemáticas histórico-locales, ese corpus de fuentes electrónicas define, dentro del universo digital que comunica al planeta, un ciberespacio propio y diferenciado, que estimula la autoafirmación comunitaria y fomenta nuevas formas de relacionamiento en la pequeña escala, a partir de redes y formatos globales (Smith y Kollock, 1999). El ciberespacio comunitario, si se lo considera en sí mismo, conforma un objeto histórico-social inédito, que ameritaría un análisis detallado. Sin embargo, lejos de transformarse en un objeto segregado y autocontenido, interactúa con las variantes más clásicas de la sociabilidad pública y privada de algunas colectividades.

Indudablemente, el uso de los sitios web como fuentes históricas y su organización en un acervo documental claramente definido plantea serios desafíos. Cabría mencionar, al respecto, el problema de la preservación de los materiales, ya que buena parte de los sitios web tienen una existencia transitoria y la inmensa mayoría experimenta modificaciones permanentes, ya que se actualizan de manera regular. En tal sentido, el desarrollo de un archivo mundial que conserve los sitios extintos y que almacene todas las modificaciones que sufren en un período concreto una herramienta de gran eficacia. Actualmente, estas funciones las cumple Internet Archive (2001), aunque no con el grado de sistematicidad que resultaría deseable.

En lo que respecta a los sitios web institucionales, especialmente si pertenecen al ámbito público, debe señalarse que contienen dos clases de cuerpos histórico-documentales de gran valor: leyes, decretos o edictos sancionados por ciertos órganos de gobierno y expedientes administrativos. En los últimos años, las autoridades nacionales y locales de decenas de países, han editado repertorios electrónicos que incorporan la totalidad de las normas jurídicas 
aprobadas a partir de una fecha específica. Asimismo, en tiempos recientes, se ha implementado formas de tramitación informática que racionalizan y dinamizan la gestión de instituciones públicas y privadas (Norris, 2007). Los expedientes reproducidos en formato electrónico (o generados desde un comienzo en dicho formato), se archivan en sitios web y el investigador accede a ellos sin que medien las dificultades características de los procedimientos tradicionales de consulta.

\subsection{Las fuentes cartográficas}

Las innovaciones tecnológicas de las últimas décadas plantean estrategias insospechadas para el estudio histórico del paisaje geográfico, transformado por la presencia humana. Ya sea que el investigador se interese por el medio rural y por las actividades económicas, sociales y culturales que lo caracterizan, o por el medio urbano en todos sus aspectos, los instrumentos digitales le permiten recopilar y reproducir viejas fuentes y generar un número ilimitado de nuevos documentos (Gregory, 2003). Con relación al relevamiento de mapas y planos, algunas colecciones testimonian la génesis y el desarrollo de una localidad específica, a partir de una secuencia de fuentes cartográficas que se publican en Internet. ${ }^{3}$ Sin embargo, los cambios más relevantes se relacionan con el diseño de mapas sobre la historia de una localidad. Así, a las clásicas representaciones en dos dimensiones se suman las que emplean tres dimensiones (cuando cartografían el paisaje en relieve) o cuatro (cuando reproducen automáticamente una secuencia de mapas en relieve que ilustran las modificaciones de dicho paisaje durante un período determinado). En cualquiera de los casos referidos, la tecnología de los así llamados Sistemas de Información Geográfica ${ }^{4}$ propicia el desarrollo de bases de datos geo-referenciales, que vinculan fenómenos representados en un mapa con una serie de documentos asociados. De este modo, el usuario selecciona un accidente geográfico o un asentamiento humano en un mapa electrónico y accede, en la base de datos correspondiente, a documentos que narran la historia del lugar escogido, o que contienen información estadística sobre él. Por último, los programas que despliegan fotografías satelitales sobre la totalidad de la superficie del planeta brindan al historiador local herramientas con las que puede crear piezas cartográficas sobre algunas de las unidades que integran su objeto de estudio, ya sea un establecimiento de campo, un complejo de viviendas, un barrio, una ciudad, un municipio o una región.

\footnotetext{
3 La David Rumsey Map Collection (2014), con 15.800 mapas digitalizados, resulta un caso típico.

4 Para una presentación detallada de la tecnología mencionada y de sus aplicaciones, véase Gregory (2003).
} 


\subsection{Las fuentes fotográficas}

Las redes telemáticas cumplen un papel relevante en las actividades de organización y difusión del patrimonio histórico-fotográfico de una comunidad. Cuando se aplican programas específicos para catalogar los fondos documentales de un archivo de materiales iconográficos, las tareas de ordenamiento y consulta se simplifican notablemente. ${ }^{5}$ Si los catálogos se publican en un sitio web institucional, se incrementan las posibilidades de que investigadores de diferentes procedencias descubran el potencial de los materiales custodiados y se interesen en ellos. Asimismo, la digitalización selectiva o exhaustiva de las piezas fotográficas de una colección pública o privada favorece la difusión mundial de sus contenidos y proyecta el patrimonio histórico local a ámbitos que lo trascienden. ${ }^{6}$

\subsection{Las fuentes orales y sonoras}

Los vínculos entre la Historia oral y la Historia local se remontan a fines del siglo XIX, cuando los investigadores comienzan a utilizar las primeras formas de registro magnetofónico como medio de preservar la memoria individual y colectiva. A partir de entonces, la recopilación de fuentes que preservan una tradición oral y la realización de entrevistas que producen -por sí mismas- nuevos documentos orales, se basan en estrategias metodológico-técnicas que responden a los cambios tecnológicos acumulativos de los últimos cien años. Más recientemente, numerosas aplicaciones informáticas, multiplicadas en cantidad y variedad, inciden de manera decisiva en las actividades relacionadas con el uso de fuentes orales, simplificando el trabajo de edición de repertorios. Ya sea que recurran al disco compacto o a los sitios web de la red mundial, proporcionan, a los integrantes de una comunidad cualquiera, registros de gran valor con respecto a su propio pasado.

Con respecto a las fuentes sonoras, el historiador, auxiliado por las nuevas tecnologías, suele desarrollar tres actividades estrechamente relacionadas: producir fuentes, recopilar y sistematizar las que ya existen y analizar, con herramientas nuevas, los materiales que se producen. Las tareas de generación de fuentes relacionadas con la Historia local obedecen a una tradición académica de larga data, que asume expresiones múltiples. De hecho, el análisis del

\footnotetext{
${ }^{5}$ Entre los utilitarios de aparición más reciente que cumple con estas funciones, se destaca Imabas 11.0 (2013).

${ }^{6}$ Existen numerosos repertorios disponibles en línea que poseen tales características. Entre ellos, Images of England (2007), que contiene 300.000 piezas iconográficas especializadas en arquitectura e infraestructura urbana y rural.
} 
universo acústico de una localidad ha estado asociado al registro de los sonidos que emanan de la vía pública, de las actividades cotidianas de diversas instituciones, de los ámbitos recreativos y de los espacios que se destinan a la sociabilidad. En lo que atañe a las fuentes musicales, es bien sabido que la recopilación de expresiones instrumentales y vocales de la música folklórica y popular, de comunidades rurales y urbanas, resulta una labor característica de historiadores, etnólogos y antropólogos a lo largo del siglo XX. Los instrumentos de grabación disponibles en la actualidad no modifican la producción y/o conservación de fuentes, sino que las simplifican notablemente, mediante un almacenamiento más eficaz y económico de los registros, y a través de la localización automática de cualquier pieza que se busque. Inclusive, algunos programas procesadores de archivos de audio detectan patrones acústicos específicos, a partir de las pautas que el usuario seleccione. De este modo, las funciones de búsqueda no solo identifican documentos, sino que también localizan fragmentos o pasajes que se corresponden con los parámetros especificados.

\subsection{Las fuentes audiovisuales}

A principios del siglo XXI, un número considerable de canales televisivos de carácter local emiten algunos de sus programas a través de Internet, y los archivan en secciones específicas de sus sitios web. De este modo, materiales audiovisuales que antes resultaban de difícil acceso comienzan a circular libremente, ${ }^{7}$ dando origen a archivos de canales televisivos comunitarios. Gracias a ellos, el investigador se informa sobre los acontecimientos que pautan la historia cotidiana de un pueblo o de una ciudad, así como las formas en que la comunidad se presenta y representa a sí misma a través de una cámara.

La incorporación en la prensa escrita digital de archivos audiovisuales multiplica las fuentes que resultan de interés al historiador. A su vez, el carácter multimediático de ciertos periódicos y de agencias de noticias diluye los límites tradicionales entre los órganos informativos y complejiza sus formatos. Aun así, la consulta pública de tales materiales y su reproducción automática y gratuita supone un avance notable en el ámbito de la investigación científico-social. La presencia en la red de medios concurrentes, que ofrecen versiones y visiones encontradas de los mismos acontecimientos, situaciones o problemas, motiva la cautela del historiador en la labor de crítica heurística de los documentos que obtiene.

\footnotetext{
7 World Wide Internet TeleVision (1998) se presenta como el ejemplo adecuado de un directorio con enlaces a más de 2700 canales de televisión que transmiten algunos contenidos de su programación en línea.
} 
En el ámbito de la producción cinematográfica, las nuevas tecnologías afectan positivamente al desarrollo de una cultura audiovisual de carácter local. Indudablemente, se trata de un desarrollo desigual, según el país y la región que se considere, así como los recursos materiales y simbólicos disponibles. En algunos casos, los historiadores vinculados con una localidad cumplen una función social y científica, al promover la organización de cinematecas o de filmotecas que recopilan la producción originada en el área. Paralelamente, gracias a la democratización del uso de los medios de registro audiovisual, un número creciente de ciudadanos documenta sus actividades diarias y los eventos más relevantes de su vida pública y privada. Esta opción, durante la mayor parte del sigo XIX resultó ser el privilegio de un reducido número de familias. En la actualidad, millones de individuos realizan filmaciones domésticas que revelan las formas cambiantes de sus rutinas en un contexto local. ${ }^{8}$ Muchos de estos registros pueden presentarse como verdaderas fuentes alternativas, ya que las grabaciones de acontecimientos políticos -e incluso militares- por parte de ciudadanos independientes, permite cuestionar las versiones que transmiten los medios masivos de comunicación, sobre todo cuando son objeto de manipulación o de censura. En tal sentido, la guerra que se libró en el Líbano entre Israel y las fuerzas de Hizbolá en julio y agosto de 2006, constituye el ejemplo de un conflicto cuyos eventos no sólo fueron registrados por los medios televisivos, sino por las cámaras de los teléfonos celulares de libaneses e israelíes que observaban, participaban o resultaban involucrados en los hechos. ${ }^{9}$ Los registros audiovisuales producidos y difundidos por Internet ilustran los estrechos vínculos entre lo local y lo global y sus consecuencias históricas.

\section{La renovación hermenéutica}

Las nuevas tecnologías aplicadas al estudio del pasado local no se limitan a la ampliación del espectro heurístico o a la sistematización de fuentes, sino que también aportan herramientas específicas para la labor hermenéutica, entendida como el procesamiento y análisis de los datos que emanan de la documentación. A modo ilustrativo, es posible referir algunos utilitarios relacionados con estrategias cualitativas y cuantitativas en materia de investigación:

\footnotetext{
${ }^{8}$ Una expresión de esta tendencia es el desarrollo de los así llamados videoblogs. Organizados como diarios personales, regularmente incorporan registros audiovisuales sobre eventos cotidianos.

9 En estas formas de registro de los hechos a partir del uso de teléfonos celulares, también resultan involucrados los mensajes de texto. El blog denominado Lebanon-Israel conflict via cellphones (2006) recopila un voluminoso material al respecto.
} 
(i) Los programas de análisis espacial. El paisaje humanizado se presenta como una de las expresiones más inmediatas y características de lo local. En tal sentido, las herramientas construidas para relevar la antropósfera en su globalidad pueden emplearse con similar eficacia en el análisis de los distintos nódulos locales del sistema socio-ecológico planetario. Así lo demuestra Google Earth (s.f.), utilitario que posibilita un abordaje holístico de todos los aspectos espaciales de las actividades políticas, económicas, sociales y culturales de las comunidades históricas.

(ii) Los programas de análisis demográfico. Para el cultivo de la Historia local, el estudio de la población resulta tan significativo como la descripción de las estructuras territoriales. El procesamiento informático de datos estadísticodemográficos que proceden de múltiples fuentes se revela como una operación esencial, ya que automatiza tareas repetitivas que requieren rigor y precisión. Las herramientas digitales que importan datos procedentes de registros censales, discriminan las cifras que se corresponden con la población local y las reordenan según los criterios y parámetros que establezca el investigador ponen de manifiesto el potencial hermenéutico de esta clase de medios. Por otra parte, la combinación de las tecnologías de análisis sociobiológico con paquetes informáticos que estructuran sus resultados ha dado origen a proyectos como Genographic (2014), en los que la Historia local y la global se fusionan en el análisis del patrimonio genético del individuo o de su grupo familiar. De este modo, cada ser humano puede reconstruir la complejísima trama que lo relaciona biológicamente con las poblaciones históricas y prehistóricas a las que pertenecieron todos sus ancestros, desde la aparición del primer hombre moderno, hace 57.000 años, hasta el más inmediato presente. Aplicado en el ámbito local, tal procedimiento evidencia la riqueza de los influjos demográficos de los que se nutre una comunidad específica. De hecho, poblaciones superficialmente homogéneas presentan una diversidad genética asombrosa, fruto del aporte de numerosos grupos, los cuales se mezclaron hasta integrar unidades culturales de gran solidez y estabilidad.

(iii) Los programas de estudios genealógicos. Se trata de un conjunto de aplicaciones representativas de los nuevos desarrollos de la Historia local, sobre todo en el contexto del universo anglosajón. Recursos como Familysearch (2012) -base de datos genealógica creada por la Iglesia de los Santos de los Últimos Días- informan detalladamente sobre las relaciones que existen entre la historia de las familias y los movimientos migratorios globales. 
Por su parte, el programa Personal Ancestral File (s.f.) proporciona algoritmos para producir complejos genogramas que describen y analizan vínculos de ascendencias, descendencia y alianzas matrimoniales entre decenas y cientos de personas.

(iv) Los programas de estudios prosopográficos. La sistematización de los datos biográficos de los integrantes de un colectivo constituye una estrategia de investigación recomendable para quienes cultivan la Historia ocal. Utilitarios que incluyen modelos básicos de biogramas, o que ya contienen fichas prosopográficas en su propio formato, facilitan las actividades de ingreso, clasificación, comparación e interpretación de insumos de tal naturaleza. ${ }^{10}$ Estas fichas originan voluminosas bases de datos que revelan, mediante operaciones automatizadas, vínculos sociales de toda índole entre los integrantes de un grupo socio-profesional, o político-institucional o políticopartidario de una localidad concreta.

(v) Los programas de análisis rético. El análisis de redes sociales, surgido en los años sesenta del pasado siglo, se orienta a la identificación de las regularidades estructurales de la acción humana en un contexto microsocial. Se trata de un enfoque que concibe a los actores como nodos interdependientes de un mismo sistema y considera a sus relaciones como canales de flujo de recursos que adoptan variadas estructuras. El análisis rético resulta revelador en contextos espacio-temporales acotados, como los que brinda la Historia ocal. Actualmente, se sirve de dos clases de programas: los que ofrecen herramientas gráficas para simbolizar las redes sociales y los que describen las relaciones que mantienen entre sí los integrantes de la red, mediante operaciones que pueden expresarse a través del lenguaje matemático o formal (Bresciano, 2005).

(vi) Los programas de análisis de contenido de texto. En la medida en que las fuentes textuales de una localidad se digitalizan paulatinamente, la aplicación de utilitarios que desagregan un documento en unidades temáticas permite descubrir lógicas expresivas y argumentales propias del contexto local. Asimismo, favorece la identificación de modismos, giros idiomáticos y contenidos léxicos diferenciales característicos del área geográfico-cultural que se aborda (Bresciano, 2005).

\footnotetext{
${ }^{10}$ Un ejemplo notable de la aplicación de las nuevas tecnologías en este campo, lo ofrece Prosopography of Anglosaxon England (2010).
} 


\section{La producción historiográfico-local en la Web}

La Historiografía local, como cualquier otra expresión del saber académico, se materializa en productos destinados a la difusión científica o a la divulgación masiva, mediante múltiples soportes y formatos. Entre las modalidades más clásicas, figuran los textos bibliográficos y los hemerográficos. La edición digital (que permite descargar la versión electrónica de un libro desde un sitio web o desde una aplicación telemática) promociona de los estudios histórico-locales, al reducir de manera notable los costos de producción y de distribución de las obras. La divulgación global, por otra parte, concede a los textos electrónicos de la Historiografía local un espacio de incidencia, de vigencia y de permanencia que nunca antes tuvieron.

En lo que respecta a la producción hemerográfica académica, la edición de revistas digitales especializadas reproduce las tendencias generales que afectan a esta clase de publicaciones. Ya sea que dispongan de una versión impresa complementaria o que solo presenten una versión electrónica, las revistas de Historia local disponen, actualmente, de sitios web que las promocionan en el ciberespacio. ${ }^{11}$ Al igual que acontece con otros medios hemerográficos de corte académico, experimentan una eclosión reciente en cuanto a su número y su variedad. El tiempo dirá si esta multiplicación y diversificación mantendrá su impulso o finalmente decantará en torno a las propuestas más estables, comprometidas con líneas y proyectos de investigación de largo plazo.

En materia de producción historiográfica institucional, los sitios web de las asociaciones de Historia local y los centros universitarios dedicados a este campo también proporcionan un cauce de socialización de trabajos de distinta enjundia. Los directorios nacionales e internacionales que sistematizan el acceso a asociaciones y centros revelan la pluralidad de ciertas trayectorias institucionales, la solidez de sus estructuras y la densidad de los vínculos capilares que desarrollan en las más pequeñas escalas. ${ }^{12}$

Existen, por otra parte, modalidades divulgativas que trascienden el espacio universitario y las fronteras tradicionales de la comunicación escrita. Se trata de aquellos sitios web que, a partir de una configuración multimediática, aportan una perspectiva caleidoscópica de las historias comunitarias. En estos sitios, la producción académica adapta su lenguaje para llegar al gran público y comparte su espacio con otras expresiones culturales centradas en la apropiación

\footnotetext{
${ }^{11}$ Sirva de ejemplo: The Local Historian. The Journal of the British Association for Local History, disponible en el sitio web de la British Association for Local History (2005).

${ }^{12}$ Véase, a modo ilustrativo, la sección "Directory of Local History and Allied Societies" del sitio web Local History On Line (2010).
} 
de pasados comunes, a través de la valoración de los patrimonios locales y la vigorización de las identidades locales. Baste como ejemplo citar el caso de la propuesta denominada Shaping San Francisco. Según sus creadores:

Shaping San Francisco is a participatory community history project documenting and archiving overlooked stories and memories of San Francisco. We are committed to defining a new kind of public space, specifically around a shared interest in our interrelated social histories. We mean "community" in a few different ways, and these definitions help shape our approach to promoting the everyday study of history.

First of all, we document and archive the stories and memories of the community. This is not how history is usually taught, and we reject the notion that there is just one way to see the events that have defined our lives. We don't accept that someone else gets to decide what those events are/were, usually favoring those who hold money and power as the agents of history. [...] We also gather oral histories from ordinary San Franciscans whose remembrances help us understand the complex fabric of life at various times in history. Some of them have dramatically affected the course of history, others have been altered by the changes happening around them, and yet others have been empowered and politicized by history as it unfolds.

This brings us to our second definition of community: that history is a participatory, creative act, a shared project of shaping our sense of life. Shaping San Francisco seeks to bring out the historian in everyone. Naturally, each individual will have a unique take on and different experience of events of which they are a part. Thus we welcome diverse contributions from the public, with their multiple perspectives, to the canon of history. [...]

Finally, we also believe in the creation of community through a shared interest in our common history. We think community arises in the effort to create a better future from the work we are doing in the present. We are committed to defining a new kind of public space, specifically around shared and interrelated social histories. (Shaping San Francisco, s.f.)

La estructura de este sitio se halla en plena consonancia con los objetivos que lo orientan, ya que contiene cinco secciones interactivas que vinculan la investigación histórica con la participación ciudadana. La primera sección, Ciclos de la Historia, presenta información sobre un calendario de tours en bicicletas que se realizan en San Francisco y que se vinculan con múltiples temáticas. La segunda sección, Excavando en la Historia, propicia las discusiones entre actores e instituciones de la sociedad civil. La tercera sección, la Web de la Historia, contiene un repositorio de documentos textuales e iconográficos de gran valor testimonial. La cuarta sección, las Páginas de la Historia, consiste en una librería virtual con obras destacadas sobre la historia de esa ciudad californiana. La quinta y última sección, Haciendo la Historia, informa sobre proyectos de 
investigación en curso. Del conjunto de estas secciones surge una concepción hipermedial e integradora, que conjuga investigación con proyección social en un formato acorde a los tiempos.

La divulgación telemática de la Historiografía local no se limita a publicaciones y proyectos. También incorpora el diseño de bibliotecas, archivos y museos virtuales de ciudades, municipios y provincias. ${ }^{13}$ De hecho, algunas propuestas actuales simulan la estructura edilicia de un museo, y de este modo presentan las piezas artístico-históricas más significativas del medio al que pertenecen. Inclusive, determinadas representaciones virtuales pueden abarcar la totalidad espacial de una comunidad concreta. Existen numerosos ejemplos de reconstrucciones virtuales de complejos arquitectónico-urbanísticos, de barrios enteros, o de una ciudad, que registran las modificaciones que todas estas unidades poblacionales experimentan a lo largo de décadas o de siglos. $^{14}$

Finalmente, cabe señalar que las nuevas tecnologías revolucionan las formas de enseñanza de la Historia local, en ámbitos formales e informales. Con respecto a los primeros, la oferta de cursos a distancia de instituciones universitarias en las que se trabaja especialmente sobre el pasado regional, motiva a algunos investigadores a continuar con su formación académica y a establecer vínculos con colegas que se interesan por sus mismos tópicos. Con relación a los ámbitos no formales, la creación de programas radiales y televisivos sobre Historia local en Internet resultan ejemplos notables del modo en que la globalización telemática puede contribuir al diálogo entre múltiples pasados.

\section{Conclusiones}

Sin lugar a dudas, Internet promueve la difusión del conocimiento histórico sobre localidades y regiones, y contribuye a una comprensión más compleja y sutil de las realidades nacionales y mundiales. Gracias a "la red de redes", los trabajos monográficos de Historia local ya no se circunscriben a los límites que establecen los medios tradicionales de divulgación. De hecho, cuando se publican en revistas digitales o en sitios web de centros universitarios, pueden convertirse en modelos referenciales para quienes llevan a cabo proyectos análogos en otros países o regiones. A su vez, el desarrollo de la Web estimula el crecimiento en cantidad y en

\footnotetext{
${ }^{13}$ Considérese el caso del sitio web Waseca Virtual History Museum (s.f.).

${ }^{14}$ Uno de los ejemplos más típicos es el que ofrece el sitio web Rome Reborn. A digital model of the Ancient Rome (2012).
} 
complejidad de las asociaciones de estudios locales, tanto las que impulsan los investigadores no profesionales como las que organizan los historiadores pertenecientes al universo académico.

El despliegue, a escala planetaria, de los medios digitales de difusión dinamiza las actividades de creación de saber, democratiza el acceso al patrimonio heurístico comunitario y diversifica las estrategias hermenéuticas. Las ediciones de fuentes locales -que suelen ser bastante onerosas cuando se realizan en formato tradicional- conceden al gran público la oportunidad de acceder a reproducciones facsimilares de documentos que antes consultaban unos pocos. Por otra parte, programas informáticos asociados a métodos novedosos transforman las prácticas de los historiadores locales, desde aquellos que se interesan por el espacio geográfico hasta los que se concentran en familias e individuos.

La circulación de los productos de la Historiografía local tampoco resulta indiferente a los cambios tecnológicos. La edición de textos académicos, la organización de publicaciones periódicas, la presencia en el ciberespacio de las entidades profesionales y los centros universitarios especializados ilustran los efectos de la aplicación de las nuevas tecnologías en este campo disciplinario. Lo mismo puede afirmarse de aquellos sitios que promueven proyectos sobre Historia local y que contienen repositorios virtuales. Inclusive la enseñanza del pasado barrial, municipal o regional resulta favorecida por la omnipresente World Wide Web. En síntesis, es posible concluir que la glocalización -en cuanto proceso característico de este tiempo- demuestra ejercer un efecto verdaderamente dinamizador en el ejercicio de una de las variantes más antiguas (y más cotidianas) de los estudios del pasado, desde que los historiadores eligen sus temas hasta que presentan sus resultados.

\section{Bibliografía citada}

AMATO, Joseph A. Rethinking Home. A case for Writing Local History. Berkeley; Los Angeles; Londres: University of California Press, 2002.

BRESCIANO, Juan Andrés. Los medios informáticos en la investigación humanística. Repertorio básico de programas y utilitarios. Montevideo: Psicolibros Waslala, 2005.

BRESCIANO, Juan Andrés. La Historiografía en el amanecer de la cultura digital. Innovaciones metodológicas, discursivas e institucionales. Montevideo: Ediciones Cruz del Sur, 2010. 
BROOKS, Pamela. How to Reseach Local History. Find out all about your house, village or town. Begbroke, Oxford: Howtobooks, 2da. ed., 2008.

BUNZ, Mercedes. The silent revolution. How digitalization transforms knowledge, work, journalism and politics without making too much noise. Basingstoke: Palgrave Macmillan, 2014.

CASTELLS, Manuel. La Era de la Información. Economía, Sociedad y Cultura. Madrid: Alianza Editorial, 1996-1998, 3 vols.

DYER, Christopher; HOPPER, Andrew; LOYD, Evelyn; TRINGHAM, Nigel (eds.). New Directions in Local History since Hoskins. Hertfordshire: University of Hertfordshire Press, 2011.

GREGORY, Ian N. A Place in History. A Guide to Use GIS in Historical Research. Oxford: Oxbow Books Limited, 2003.

KAMMEN, Karol. On Doing Local History. Walnut Creek; Lanham; Nueva York; Oxford: Altamira Press, 2da ed., 2003.

KYVIG, David E.; MARTY, Myron A. Nearby History. Exploring the Past Around You. Lanham; Nueva York; Toronto; Plymouth: Altamira Press, 3ra. ed., 2010.

NORRIS, Donald F. (ed.). Current issues and trends in e-government research. Hershey: Cybertech Pub., 2007.

PATEL, Aleesha. The Survival of the Newspaper in the Digital Age of Communication, [En línea]. Disponible en Internet en: http://www.stern.nyu.edu/sites/default/files/assets/documents/con_043010.pdf, 2010.

ROBERTSON, Ronald. Globalization. Social Theory and Global Culture. Londres: Sage, 1992.

SMITH, Marc A.; KOLLOCK, Peter (eds.) Communities in Cyberspace. Londres; Nueva York: Routledge, 1999. 


\section{Fuentes telemáticas citadas}

David Rumsey Map Collection [En línea]. Disponible en Internet en http://www.davidrumsey.com, 2014.

"Directory of local history and allied societies" en Local History On Line. [En línea]. Disponible en Internet en http://www.local-history.co.uk/Groups, 2010.

Familysearch.org. [En línea]. Disponible en Internet en https://familysearch.org/

Google Earth. [En línea]. Disponible en Internet en https://www.google.com/earth, s.f.

“Imabas11.0” en Pixandmore. Digital Image Management. [En línea]. Disponible en Internet en http://www.pixandmore.com, 2013.

Images of England. [En línea]. Disponible en Internet en http://www.imagesofengland.org.uk, 2007.

Internet Archive. Universal Access to All Knowledge. [En línea]. Disponible en Internet en https://archive.org/, 2001.

Lebanon-Israel Conflict Via Cellphones. [En línea]. Disponible en Internet en http://itf.typepad.com/lebanon, 2006.

Online Books. [En línea]. Disponible en Internet en http://webbooksmanager.com, 2014.

“Personal Ancestral File” en Softonic. [En línea]. Disponible en Internet en http://personalancestral-file.softonic.com, s.f.

Prosopography of Anglosaxon England. [En línea]. Disponible en Internet en http://www.pase.ac.uk/index.html, 2010.

Questia. Trusted On Line Research. [En línea]. Disponible en Internet en http://www.questia.com, 2014.

Rome Reborn. A Digital Model of the Ancient Rome. [En línea]. Disponible en Internet en http://romereborn.frischerconsulting.com, 2012.

Shaping San Francisco. [En línea]. Disponible en Internet en: Qnline Books. [En línea] Disponible en Internet en http://www.shapingsf.org/, s.f. 
The Genographic Project. [En línea]. Disponible en Internet en https://genographic.nationalgeographic.com, 2014.

"The Local Historian. The Journal of the British Association for Local History" en British Association for Local History. [En línea]. Disponible en Internet en http://www.balh.co.uk/tlh/index.html, 2005.

Waseca Virtual History Museum, [En línea]. Disponible en Internet en http://www.waseca.k12.mn.us/vhm/index.html, s.f.

World Wide Internet TeleVision [En línea]. Disponible en Internet en http://wwitv.com, 1998. 